\title{
Review
}

Johannes M. Herrmann* and Jan Riemer*

\section{Apoptosis inducing factor and mitochondrial NADH dehydrogenases: redox-controlled gear boxes to switch between mitochondrial biogenesis and cell death}

https://doi.org/10.1515/hsz-2020-0254

Received July 15, 2020; accepted August 3, 2020; published online August 25, 2020

\begin{abstract}
The mitochondrial complex I serves as entry point for NADH into the electron transport chain. In animals, fungi and plants, additional NADH dehydrogenases carry out the same electron transfer reaction, however they do not pump protons. The apoptosis inducing factor (AIF, AIFM1 in humans) is a famous member of this group as it was the first pro-apoptotic protein identified that can induce caspase-independent cell death. Recent studies on AIFM1 and the NADH dehydrogenase Nde1 of baker's yeast revealed two independent and experimentally separable activities of this class of enzymes: On the one hand, these proteins promote the functionality of mitochondrial respiration in different ways: They channel electrons into the respiratory chain and, at least in animals, promote the import of Mia40 (named MIA40 or CHCHD4 in humans) and the assembly of complex I. On the other hand, they can give rise to pro-apoptotic fragments that are released from the mitochondria to trigger cell death. Here we propose that AIFM1 and Nde1 serve as conserved redox switches which measure metabolic conditions on the mitochondrial surface and translate it into a binary life/death decision. This function is conserved among eukaryotic cells and apparently used to purge metabolically compromised cells from populations.
\end{abstract}

Keywords: cell death; complex I; MIA40; mitochondria; protein import; redox.

\footnotetext{
*Corresponding authors: Johannes M. Herrmann, Department of Cell Biology, University of Kaiserslautern, Erwin-Schrödinger-Strasse 13, D-67663 Kaiserslautern, Germany,

E-mail: hannes.herrmann@biologie.uni-kl.de; and Jan Riemer, Department of Biochemistry, University of Cologne, Zülpicher Str. 47A, D-50674 Cologne, Germany, E-mail: jan.riemer@uni-koeln.de. https://orcid.org/0000-0003-2081-4506 (J.M. Herrmann).
}

Introduction

The strange case of Dr. Jekyll and Mr. Hyde is a famous novel by Robert Louis Stevenson about a person of remarkable dual nature: Dr. Jekyll is a doctor with amiable personality who helps others and has many friends. But sometimes, he converts into Mr. Hyde who is shockingly evil and brings death to others. Several mitochondrial NADH dehydrogenases are likewise of dual nature. They are enzymes of the respiratory chain and, at least in animal cells, promote mitochondrial biogenesis. However, under certain conditions they can convert into pro-apoptotic factors that induce irreversible DNA fragmentation and cell death.

The mitochondrial respiratory chain utilizes the flow of electrons from NADH to oxygen to pump protons into the IMS. The return of these protons to the matrix is used to generate ATP. The capacity of this system is impressive. In a single human being, about $60 \mathrm{~kg}$ of ATP are produced in a single day, by far most of these in mitochondria. Despite the huge amount of the end product ATP, the substrates of the respiratory chain, oxygen and $\mathrm{NADH}$, are often not limiting in well-fed organisms. On the contrary, high NADH levels and correspondently, low levels of $\mathrm{NAD}^{+}$, are not beneficial but associated with pathological conditions and premature aging (Katsyuba et al. 2018). Cells employ alternative pathways to oxidize surplus NADH and NADPH, such as the production of lactate from pyruvate or that of $\alpha$-hydroxybutyrate from $\alpha$-ketobutyrate or acetoacetate (Thompson Legault et al. 2015). Many organisms use mitochondrial enzymes that bypass the respiratory chain, i.e., they transport electrons but do not pump electrons. Since these enzymes are running idle they have the potential to quickly and efficiently reduce the NAD(P)H redox potential. $\mathrm{NAD}(\mathrm{P}) \mathrm{H}$ dehydrogenases are shortcuts that bypass complex I (from NADH to ubiquinone) and alternative oxidases act downstream of ubiquinone to bypass complexes III and/or IV. These enzymes are ubiquitously 
present in plants and fungi, but are also found in some animals where they can play a role in thermogenesis (Bertsova et al. 2004; Nguyen et al. 2020): In humans, brown adipose cells are specialized in heat production. They dissipate energy via the uncoupling protein UCP1 which serves as proton leak in the inner membrane. In addition to UCP1, these cells express AIFM2, a special isoform of an NADH dehydrogenase, to speed up electron flow through the respiratory chain. AIFM2 and its more ubiquitously expressed homolog AIFM1 presumably play a so far poorly analyzed physiological role in metabolism in many cells. Recent studies suggests that these enzymes are part of an OXPHOS paralleling system that reduces the risk of phospholipid peroxidation and ferroptosis, although this system is still poorly characterized (Bersuker et al. 2019; Doll et al. 2019). Despite this 'life-saving' function as ferroptosis suppressor, apoptosis inducing factors were initially identified as factors that can trigger a unique, caspase-independent apoptotic program.

In this review we will discuss these different functions of mitochondrial NADH dehydrogenases on the basis of recent discoveries made on mammalian and fungal isoforms.

\section{NADH dehydrogenases, a conserved group of enzymes}

Alternative (or type II) NADH dehydrogenases are single or oligo subunit enzymes which catalyze the same reaction as complex I but they do not pump protons across the inner membrane (Figure 1A). Unlike complex I, they lack flavin mononucleotide (FMN) and iron-sulfur clusters as reactive groups. Instead, they contain a flavin adenine dinucleotide (FAD) cofactor to mediate the electron transfer from NAD(P) $\mathrm{H}$ to ubiquinone. While complex I is deeply integrated into the inner membrane, alternative NADH dehydrogenases are not membrane-embedded, but rather associated to the membrane surface, either on the IMS side (external NADH dehydrogenases) or the matrix side (internal NADH dehydrogenases). They often are firmly attached to the membrane (this is where ubiquinone serves as their electron acceptor) by N-terminal transmembrane anchors or/and by a C-terminal hydrophobic membrane-binding region. NADH dehydrogenases belong to a large group of oxidoreductases sharing the Rossmann fold (Mate et al. 2002; Rossmann and Argos, 1978; Sorrentino et al. 2015, 2017). This group also includes enzymes such as thioredoxin reductase, dihydrolipamide dehydrogenase (the E3 component of pyruvate dehydrogenase), sulfide:quinone oxidoreductase and flavin-dependent monooxygenases (e.g., Coq6 in yeast).
Alternative NADH dehydrogenases are ubiquitously expressed in fungi and plants (Figure 1B). In baker's yeast, three homologues are well characterized: two external (Nde1 and Nde2) and one internal (Ndi1) isoforms (Luttik et al. 1998; Saladi et al. 2020). Since baker's yeast has no complex I, they serve as predominant entry sites for NADH electrons into the respiratory chain. Thereby, Nde1 and Nde2 presumably accept NADH that is synthesized by glycolysis in the cytosol and Ndi1 electrons produced by the TCA cycle in the matrix, however, all three enzymes are functionally redundant. Whereas single mutants (in
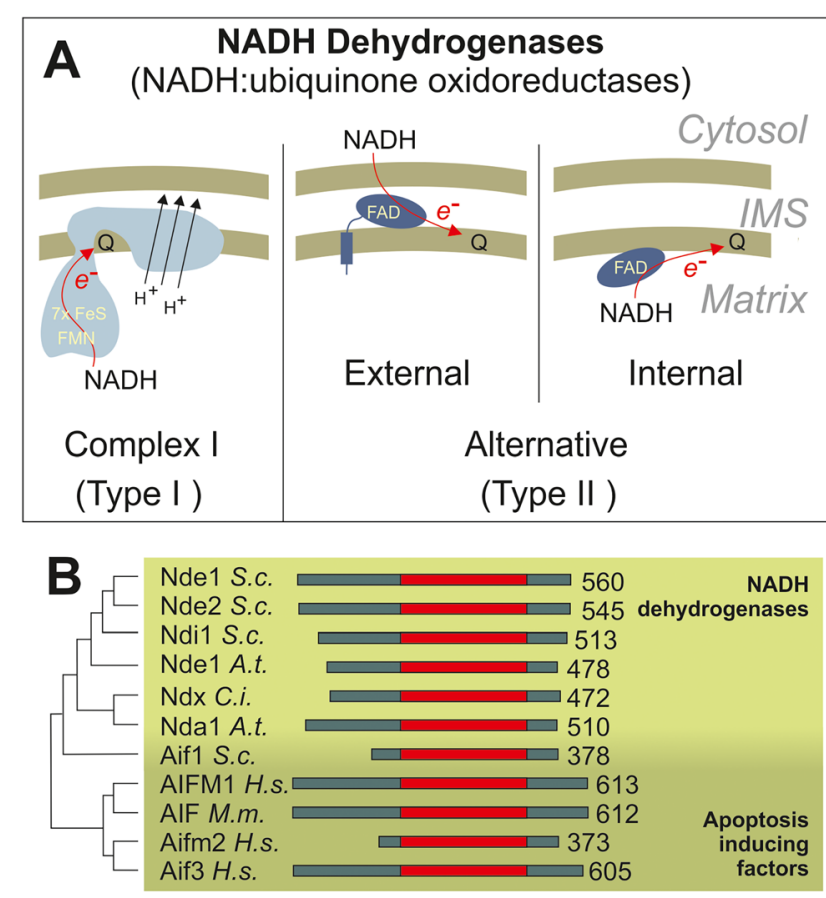

Figure 1: NADH dehydrogenases transfer electrons from $N A D(P) H$ to ubiquinone. (A) In most organisms, the mitochondrial complex I serves as the sole or predominant entry point for electrons into the respiratory chain. However, external or internal NADH dehydrogenases provide additional, alternative electron transfer routes. These structurally rather simple flavoproteins do not pump electrons. They are ubiquitously found in fungi and plants, but also in some animals. Baker's yeast lost complex I and uses exclusively alternative NADH dehydrogenases for respiration. Q, ubiquinone. (B) Structural overview of NADH dehydrogenases and apoptosis inducing factors. All these proteins share the FAD- and $\mathrm{NADH}$-binding region (shown in red) that are characterized by conserved Rossmann folds. The flanking regions are more variable, however, they consistently present matrix-targeting signals on their $\mathrm{N}$-termini followed, in the case of external dehydrogenases and apoptosis inducing factors, by membrane-anchoring hydrophobic regions. The sequences shown are that of Nde1, Nde2, Ndi1 and Aif1 of baker's yeast (S.c.), Nde1 and Nda1 of Arabidopsis thaliana (CAA18713.1 and At1g06820), Ndx of C. intestinalis (XP_002122465. 1), AIFM1, AIFM2 and AIF2 of Homo sapiens (NP_004199.1, AAH23601.1, NP_653305.1) as well as of murine AIF (NP_036149.1). 
particular $\Delta$ ndi1) show mild respiration defects (Marres et al. 1991), triple deletion mutants in which all three enzymes are deleted are unable to grow on non-fermentable carbon sources. Aif1 is a fourth member of this family. It shows the highest similarity to the human AIFM1 and a function in electron transport is not unambiguously demonstrated, though appears likely (Wissing et al. 2004). Yeast mutants lacking Aif1 show a synthetic growth defect in the absence of Gsh1, the enzyme that carries out the first step in glutathione synthesis (Costanzo et al. 2010). This points to a physiological role of Aif1 in redox homeostasis, in line with a function of Aif1 in NAD(P)H metabolism.

Alternative NADH dehydrogenases were also found in many animals, in particular in sessile marine organisms (Matus-Ortega et al. 2011; McDonald and Gospodaryov, 2019). These enzymes are presumably not required for growth at the hypoxic conditions of these life forms, however, they might serve as important valves on the respiratory chain when oxygen tensions suddenly rise. This hypothesis is supported by experiments in Drosophila melanogaster (which normally lacks alternative NADH dehydrogenases): expression of the enzyme of the tunicate Ciona intestinalis in flies provides resistance to specific stresses and can prolong life (Gospodaryov et al. 2020).

It is likely, that apoptosis inducing factors play a similar 'physiological' role in cellular redox metabolism (Cheung et al. 2006; Elguindy and Nakamaru-Ogiso, 2015; Miramar et al. 2001). In mice, AIF is essential and homozygous mutants are embryonically lethal (Joza et al. 2001). Heterozygous deletion mutants (so-called harlequin mice) are viable but show severe defects in mitochondrial functionality (Coughlan et al. 2016). In humans, mutations in the AIFM1 locus on the $\mathrm{X}$ chromosome (at position Xq26.1) lead to diseases that show symptoms such as mitochondrial encephalomyopathy, axonal sensorimotor neuropathy, ataxia or deafness. These diseases are phenotypically similar to those caused by mutations in other mitochondrial components. They include syndromes such as the Combined Oxidative Phosphorylation Deficiency 6 (Ghezzi et al. 2010), $X$-linked recessive Charcot-Marie-Tooth disease4 or Cowchock syndrome (Rinaldi et al. 2012), and X-linked Deafness 5 (Zong et al. 2015). These defects might in part be due to the role of AIFM1 in the biogenesis of complex I and the mitochondrial disulfide relay system (see below), but presumably also due the physiological relevance of AIFM1 in electron transport from NADH (and to a lower degree NADPH) to ubiquinone. However, since the different paths by which electrons are funneled into the respiratory chain are highly redundant, the individual share of each enzyme in electron flux under physiological conditions is not known.

\section{AIFM1, a partner of MIA40 in the mitochondrial disulfide relay}

AIFM1 resides in the mitochondrial IMS. It is synthesized on cytosolic ribosomes as a 613 amino acid long precursor protein. Its import into the IMS depends on an N-terminal bipartite mitochondrial targeting sequence (AIFM1-MTS) (Susin et al. 1999). The AIFM1-MTS drives the AIFM1 precursor across the mitochondrial outer membrane and anchors it in a translocase of the inner mitochondrial membrane (TIMM23)-dependent manner in the inner mitochondrial membrane. There, the matrix-localized presequence of the AIFM1 precursor is removed by the matrix processing peptidase MPP resulting in the mature membrane bound form of AIFM1 that lacks the first 54 amino acid residues compared to the precursor (Otera et al. 2005).

Apart from its role in apoptosis induction, AIFM1 has been linked to assembly and maintenance of respiratory chain complexes. Human and mouse cells lacking AIFM1 exhibited reduced levels and activity of complex I (Kollias et al. 1992; Troulinaki et al. 2018; Urbano et al. 2005; Vahsen et al. 2004). Work in other model organisms such as Saccharomyces cerevisiae, D. melanogaster and Caenorhabditis elegans supported a role of AIFM1 in respiratory chain assembly and maintenance. Deletion of the AIFM1 homolog AIF1 in yeast resulted in altered growth on nonfermentable carbon sources like lactate and glycerol (Vahsen et al. 2004). AIF knockout in flies led to larval lethality due to loss of complex I and complex IV activities (Joza et al. 2008). Likewise, downregulation of the C. elegans homolog wah-1 decreased mitochondrial respiration (Troulinaki et al. 2018).

AIFM1 is no subunit of complex I and its molecular role in respiratory chain complex assembly is not fully understood. AIFM1 is not influencing the expression of OXPHOS genes (Vahsen et al. 2004) pointing to a posttranslational role of AIFM1 during respiratory chain assembly. Indeed, AIFM1 plays an important but indirect role for the import of respiratory chain subunits into the IMS as it facilitates the import of the oxidoreductase MIA40 (also CHCHD4) (Hangen et al. 2015; Meyer et al. 2015; Petrungaro et al. 2015). Owing to this functional connection, MIA40 and AIFM1 share very similar gene essentiality profiles in systematic CRISPR knockout studies (DepMap portal) (Dempster et al. 2019), supporting their relevance for the same cellular processes.

The mitochondrial disulfide relay is a machinery that mediates protein import into the IMS and concomitant oxidative protein folding (Edwards et al. 2020; Finger and Riemer, 2020; Fischer et al. 2013; Mesecke et al. 2005; Peleh 
et al. 2017; Riemer et al. 2015; Rissler et al. 2005). It relies on the oxidoreductase MIA40 (also CHCHD4) which introduces disulfide bonds into newly imported IMS proteins. In addition, MIA40 serves as import receptor and binding site for substrates coming from the cytosol through the TOM channel. Using its oxidoreductase and chaperone activity, MIA40 catalyzes the oxidative folding of proteins and their insertion into different protein complexes in the IMS and the inner membrane (Erdogan et al. 2018; Habich et al. 2019; Okamoto et al. 2014; Peleh et al. 2016; Ramesh et al. 2016; Wrobel et al. 2016).

Yeast Mia40 contains an N-terminal bipartite mitochondrial targeting sequence that guides the protein into the IMS on the matrix-targeting pathway (Backes and Herrmann, 2017; Callegari et al. 2020; Mokranjac, 2020). Such a signal is lacking in MIA40 of animals. Instead, human MIA40 contains an N-terminal sequence of 40 residues which binds to an AIFM1 dimer that forms in the presence of NADH (Hangen et al. 2015); this interaction apparently drives the translocation of MIA40 through the translocase of the outer membrane (TOM) complex though details are not entirely clear (Figure 2). Removal of the $\mathrm{N}$-terminal amino acids from MIA40 or the depletion of AIFM1 (by siRNA) strongly impairs MIA40 accumulation in the IMS. This reduction in mitochondrial MIA40 levels is also observed in the harlequin mouse model, however, there exists a surprising and unexplained heterogeneity in different tissues of the mice (Meyer et al. 2015; Wischhof et al. 2018).

Since MIA40 imports several complex I subunits (Friederich et al. 2017), the relevance of AIFM1 for complex I biogenesis is explained by the AIFM1-dependent MIA40 biogenesis. Accordingly, when MIA40 is overexpressed or imported as fusion with a bipartite presequence in an AIFM1-independent manner, AIFM1 was found to be largely dispensable for complex I biogenesis (Hangen et al. 2015; Meyer et al. 2015).

The substrates of the disulfide relay significantly vary in their dependence on MIA40 levels (Habich et al. 2019) and, again, strong differences among different tissues were described. For example, a patient expressing a cysteine mutant of the MIA40 substrate NDUFB10, a structural subunit of complex I, had no detectable NDUFB10 protein in his liver and muscle but normal levels in fibroblasts (Friederich et al. 2017).

Thus, in mammalian cells, alternative NADH dehydrogenases serve as important, though not necessarily essential import receptors of MIA40. Here, mitochondria utilize the NADH-dependent interaction of AIFM1 and MIA40 to regulate the biogenesis of complex I subunits in dependence of the prevailing $\mathrm{NADH}$ concentrations.

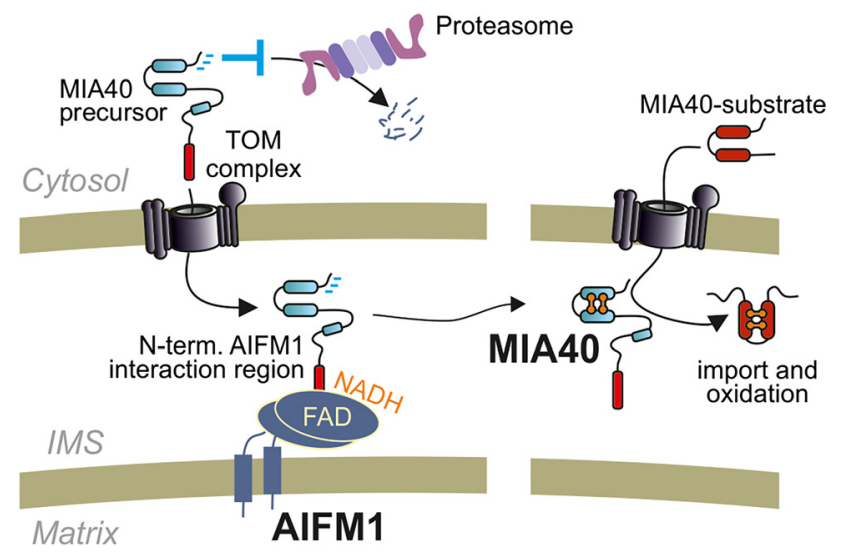

Figure 2: AIFM1 facilitates the import of MIA40, and indirectly that of complex I subunits into the IMS of mammalian cells. In humans, MIA40 carries an $\mathrm{N}$-terminal stretch of 40 residues (shown in red) that serves as binding site for AIFM1. NADH-dependent dimerization of AIFM1 is a prerequisite for AIFM1 binding and, hence, the efficient import and oxidative folding of MIA40. Once imported and oxidized MIA40 drives the import and oxidation of its substrates. Nonimported MIA40 is stable in the cytosol as its C-terminal negatively charged regions prevents proteasomal degradation.

Apparently, mammalian mitochondria added this regulatory step to the MIA40 import pathway to allow its control by the metabolic conditions.

\section{AIFM1/Nde1 can induce apoptosis}

AIFM1 was initially identified as a mitochondrial protein that, to induce apoptosis, is released into the cytosol, from where it translocates into the nucleus in order to initiate DNA fragmentation and cell death (Susin et al. 1996, 1999). This pro-apoptotic role of AIFM1 is described in depth in several excellent review articles (e.g., Bano and Prehn 2018; Dawson and Dawson 2017; Hangen et al. 2010a; Norberg et al. 2010b). Like other factors that are relevant for apoptosis, such as cytochrome $c$, SMAC/Diablo and Htra2/ Omi, AIFM1 is a protein of the IMS and initially it was proposed that in order to induce cell death, the outer membrane is ruptured to release a detrimental cocktail of killer proteins. This view is presumably too simplistic and incorrect. Rather, mitochondrial proteins are released into the cytosol in a more specific fashion which includes processes such as Bax/Bak-mediated outer membrane permeabilization.

AIFM1 is not involved in the 'canonical', caspasedependent apoptosis program. Its role in pro-apoptotic signaling therefore is distinct from that of cytochrome $c$ and caspases. Moreover, the release of AIFM1 from mitochondria is not triggered by a mechanical rupturing of the 
outer membrane (that would spill the content of the IMS into the cytosol). Rather, the release of pro-apoptotic factors from the IMS into the cytosol, and in particular that of AIFM1, can occur in a well-controlled and specific manner which, despite many years of intensive research, is still not very well understood (Pena-Blanco and GarciaSaez 2018).

The classical model for the release of AIFM1 from mitochondria (Figure $3 \mathrm{~A}$ ) proposes its calpain-mediated cleavage $\mathrm{C}$-terminally of its membrane anchor in a calcium and oxidation-triggered reaction (Norberg et al. 2010a; Ozaki et al. 2008; Polster et al. 2005). Calpains are calciumactivated cysteine proteases. In mammalian cells, several isoforms exist which are present in the cytosol as well as in the matrix and the IMS of mitochondria. The relevance of calpain for AIFM1 cleavage in vivo was questioned in several studies. Nonetheless, at least in vitro, the addition of calpains to inner membrane vesicles leads to the generation of the identical G102 $\downarrow$ L103 pro-apoptotic AIFM1 fragment that induces cell death in vivo (Polster et al. 2005). Moreover, inhibitory peptides that block the activity of calpains can protect rat retinal ganglion cells from AIFM1-dependent ischemia/reperfusion injury (Ozaki et al. 2009). However, in this context, it is very difficult to disentangle the molecular functions of AIFM1 unambiguously since it is involved in complex I assembly, in mitochondrial redox regulation and in pro-apoptotic signaling, thus in three processes that are directly relevant in the context of ischemia/reperfusion injury (Chen et al. 2019; Thompson et al. 2016).
A recent study in yeast proposed an alternative process by which the pro-apoptotic cytosolic fragment is generated (Figure 3B). The yeast NADH dehydrogenase Nde1 has a topology similar to that of AIFM1. It is integrated into the inner membrane by an $\mathrm{N}$-terminal membrane anchor and exposes an oxidoreductase domain into the IMS. However, there is a considerable fraction of the protein which has a different topology as it exposes its oxidoreductase domain into the cytosol (Saladi et al. 2020; Zahedi et al. 2006). This species is generated by an incomplete translocation across the outer membrane. If the $\mathrm{N}$ terminal targeting segment of Nde1 is replaced by the 'strong' import sequence of the inner membrane protein Mia40, only the IMS species is generated. Thus, the Nde1 targeting sequence obviously generates on purpose two versions: an IMS-located form which serves as electron transfer protein from NADH to ubiquione, and a cytosol-exposed form which is catalytically inactive (since it is inaccessible to ubiquinone). Interestingly, this surface-exposed form is highly toxic and induces apoptosis in yeast cells. Under pro-apoptotic conditions, such as in the presence of acetic acid, it gives rise to a cytosolic fragment that is under control of the mitochondrial i-AAA protease and the proteasome system (Augustin et al. 2005; Gomes et al. 2013; Saladi et al. 2020). Such pro-apoptotic fragments can presumably also be generated from other NADH dehydrogenases in yeast such as Ndi1 or Aif1 (Cui et al. 2012; Li et al. 2006; Muzaffar and Chattoo 2017; Wissing et al. 2004).

The similarity of the Nde1-mediated apoptosis in yeast and the AIFM1-dependent cell death in animal cells is very

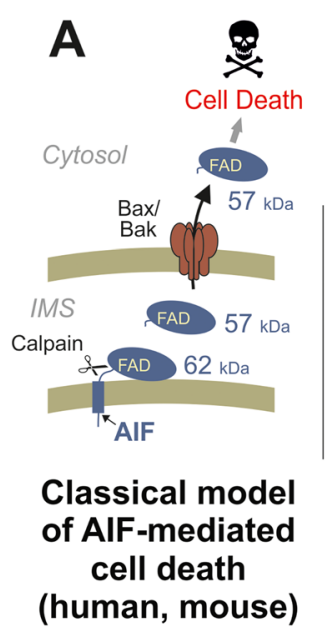

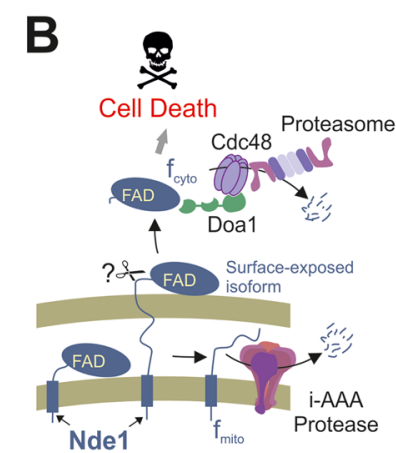
Nde1-mediated cell death (yeast)

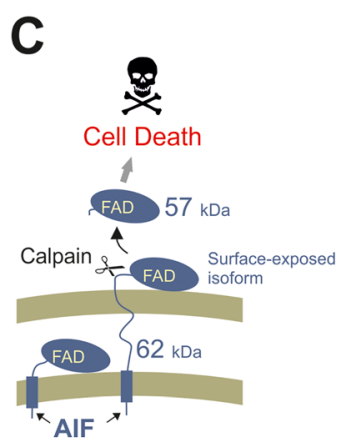
Alternative model of AIF-mediated cell death

Figure 3: Pro-apoptotic signaling by $\mathrm{NADH}$ dehydrogenases. (A) According to the classical model, AIFM1 resides within the IMS. Upon induction of apoptosis, the calcium- and redox-regulated protease calpain releases a $57 \mathrm{kDa}$ fragment from the inner membrane. This fragment is translocated through the outer membrane by the pro-apoptotic BCL-2 family effector proteins Bax/Bak (Pena-Blanco and Garcia-Saez 2018) into the cytosol from where it enters the nucleus to bind DNA. DNA fragmentation and chromatin condensation then initiate the irreversible cell death. (B) Nde1 forms two distinct topomers, one in the IMS and one exposed to the cytosol. The latter gives rise to two fragments ( $f_{\text {mito }}$ and $f_{\text {cyto }}$ )

which are under control of the i-AAA protease and the proteasome system, respectively. The fcyto-Fragment is particularly enriched in mutants with compromised mitochondria were the membrane potential is low (Saladi et al. 2020). It can efficiently induce cell death, for example in the presence of acetic acid or in aging cells. (C) An alternative model of AIFM1-mediated cell death proposes the generation of the $57 \mathrm{kDa}$ fragment from a surface-exposed topomer. It should be noted that this alternative model and the classical Bax/Bad-depending process are not mutually exclusive and might be of relevance under different conditions or in different cell types. 
striking, suggesting that the underlying principles are well conserved among eukaryotes. However, the mechanism by which the pro-apoptotic fragment is formed appears to be entirely different: In animals, the cleavage was proposed to produce the fragment initially in the IMS from where it is translocated across the outer membrane by Bax/Bak complexes or by mitochondrial permeability transition pores (Bidere et al. 2003; Moubarak et al. 2007). In contrast, the pro-apoptotic fragment of Nde1 is generated on the mitochondrial surface in the cytosol from a minor topomer, so that a translocation across the outer membrane is not required. Is it possible, that such an alternative mechanism (Figure 3C) is also of relevance in the context of AIFM1mediated cell death? Indeed, a surface-exposed topomer of AIFM1 was described for neurons and it was proposed that this species gives rise to the pro-apoptotic fragment $(\mathrm{Yu}$ et al. 2009). Moreover, more recent studies on mouse models (Engel et al. 2010) challenge the initial in vitro data from which a critical relevance of proapoptotic Bcl-2 proteins such as Bid for AIFM1 translocation across the outer membrane were concluded. Thus, a minor surface-exposed topomer of AIFM1 might also exist in humans and be of direct relevance for the pro-apoptotic function of AIFM1. Since the IMS-localized form is predominant, such a 'moonlighting' second species might be difficult to identify biochemically. Immunoprecipitation and proximity labeling, however, did identify AIFM1 as an interactor of parkin on the mitochondrial surface, in line with the existence of a cytosol-exposed species (Guida et al. 2019). This species might be controlled by the proteasome (Liu et al. 2010) in the same way as the surface-exposed Nde1 topomer of yeast cells.

Interestingly, neurons express an alternative AIFM1 variant: AIF2 is a brain-specific isoform generated by alternative splicing (use of exon 2b) (Hangen et al. 2010b). This isoform is identical to the canonical AIFM1 except for the N-terminal transmembrane region which serves as stop-anchor sequence. It was proposed that the different properties of this membrane anchor bind the protein more tightly to the inner membrane explaining why AIF2 favors its physiological and disfavors its pro-apoptotic potential (Hangen et al. 2010b). This physiological function as an NADH dehydrogenase in an electron transfer route that parallels the respiratory chain is also supported by recent studies that showed that AIF2 reduces respiration-induced lipid peroxidation and ferroptosis (Bersuker et al. 2019; Doll et al. 2019). In its properties, AIF2 is very reminiscent to the Mia40-Nde1 fusion protein of yeast cells which prevented the formation of the surface-exposed topomer and therefore could not trigger cell death (Saladi et al. 2020).

\section{Mitochondrial oxidoreductases as redox-controlled switches to commit life/death decisions}

NADH dehydrogenases obviously have different conserved functions: (1) they serve as redox enzymes that bypass the respiratory chain. This valve-like role on the respiratory chain is particularly important during unstable oxygen conditions such as in marine organisms or in the context of pathological ischemia-reperfusion injury, but also relevant to prevent oxidative damage in mitochondria under physiological conditions. (2) NADH dehydrogenases serve as 'apoptosis inducing factors' in a poorly understood process that involves the formation of a toxic protein fragment on the outside or inside of the mitochondrial outer membrane. (3) In animals but not in yeast, AIFM1 serves as biogenesis factor for MIA40 and thus, indirectly, for many IMS proteins. Why did Nature entrust one protein with these seemingly unrelated functions? As valves on the respiratory chain, NADH dehydrogenases serve as sensors of the metabolic fluxes in mitochondria. It appears likely that this metabolic information is crucial to control their role as proapoptotic component as well as their function as MIA40 import receptor. According to this hypothesis, alternative NADH dehydrogenases serve as regulatory metabolic hubs on the mitochondrial surface that can exhibit "Dr. Jekyll and Mr. Hyde"-like activities depending on the local prevailing physiological conditions. This regulation of these exciting redox controlled gear boxes on the mitochondrial surface will have to be studied in more depth in the future.

Acknowledgments: This work was supported by the priority program (Schwerpunktprogramm SPP 1710): Dynamics of Thiol-based Redox Switches in Cellular Physiology of the Deutsche Forschungsgemeinschaft (DFG). Both authors are grateful for the DFG funding. This review serves as final report for the grants to J.R. and J.M.H. Author contribution: All the authors have accepted responsibility for the entire content of this submitted manuscript and approved submission.

Research funding: This research was supported by the Deutsche Forschungsgemeinschaft (DFG) (Schwerpunkt programm SPP 1710).

Conflict of interest statement: The authors declare no conflicts of interest regarding this article.

\section{References}

Augustin, S., Nolden, M., Muller, S., Hardt, O., Arnold, I., and Langer, T. (2005). Characterization of peptides released from 
mitochondria: evidence for constant proteolysis and peptide efflux. J. Biol. Chem. 280: 2691-2699.

Backes, S., and Herrmann, J.M. (2017). Protein translocation into the intermembrane space and matrix of mitochondria: mechanisms and driving forces. Front. Mol. Biosci. 4: 83.

Bano, D., and Prehn, J.H.M. (2018). Apoptosis-inducing factor (AIF) in Physiology and disease: the tale of a repented natural born killer. eBio Med. 30: 29-37.

Bersuker, K., Hendricks, J.M., Li, Z., Magtanong, L., Ford, B., Tang, P.H., Roberts, M.A., Tong, B., Maimone, T.J., Zoncu, R., et al. (2019). The CoQ oxidoreductase FSP1 acts parallel to GPX4 to inhibit ferroptosis. Nature 575: 688-692.

Bertsova, Y.V., Popov, V.N., and Bogachev, A.V. (2004). NADH oxidation by mitochondria from the thermogenic plant Arum orientale. Biochemistry 69: 580-584.

Bidere, N., Lorenzo, H.K., Carmona, S., Laforge, M., Harper, F., Dumont, C., and Senik, A. (2003). Cathepsin D triggers Bax activation, resulting in selective apoptosis-inducing factor (AIF) relocation in T lymphocytes entering the early commitment phase to apoptosis. J. Biol. Chem. 278: 31401-31411.

Callegari, S., Cruz-Zaragoza, L.D., and Rehling, P. (2020). From TOM to the TIM23 complex - handing over of a precursor. J. Biol. Chem. 401: 709-721.

Chen, Q., Thompson, J., Hu, Y., Dean, J., and Lesnefsky, E.J. (2019). Inhibition of the ubiquitous calpains protects complex I activity and enables improved mitophagy in the heart following ischemia-reperfusion. Am. J. Cell. Physiol. 317: C910-C921.

Cheung, E.C., Joza, N., Steenaart, N.A., McClellan, K.A., Neuspiel, M., McNamara, S., MacLaurin, J.G., Rippstein, P., Park, D.S., Shore, G.C., et al. (2006). Dissociating the dual roles of apoptosisinducing factor in maintaining mitochondrial structure and apoptosis. EMBO J. 25: 4061-4073.

Costanzo, M., Baryshnikova, A., Bellay, J., Kim, Y., Spear, E.D., Sevier, C.S., Ding, H., Koh, J.L., Toufighi, K., Mostafavi, S., et al. (2010). The genetic landscape of a cell. Science 327: 425-431.

Coughlan, M.T., Higgins, G.C., Nguyen, T.V., Penfold, S.A., ThallasBonke, V., Tan, S.M., Ramm, G., Van Bergen, N.J., Henstridge, D.C., Sourris, K.C., et al. (2016). Deficiency in apoptosis-inducing factor recapitulates chronic kidney disease via aberrant mitochondrial homeostasis. Diabetes 65: 1085-1098.

Cui, Y., Zhao, S., Wu, Z., Dai, P., and Zhou, B. (2012). Mitochondrial release of the NADH dehydrogenase Ndi1 induces apoptosis in yeast. Mol. Biol. Cell 23: 4373-4382.

Dawson, T.M., and Dawson, V.L. (2017). Mitochondrial mechanisms of neuronal cell death: potential therapeutics. Annu. Rev. Pharmacol. Toxicol. 57: 437-454.

Dempster, J.M., Rossen, J., Kazachkova, M., Pan, J., Kugener, G., Root, D.E., and Tsherniak, A. (2019). Extracting biological insights from the Project Achilles Genome-Scale CRISPR screens in cancer cell lines. bioRxiv 720243. https://doi.org/10.1101/720243.

Doll, S., Freitas, F.P., Shah, R., Aldrovandi, M., da Silva, M.C., Ingold, I., Goya Grocin, A., Xavier da Silva, T.N., Panzilius, E., Scheel, C.H., et al. (2019). FSP1 is a glutathione-independent ferroptosis suppressor. Nature 575: 693-698.

Edwards, R., Gerlich, S., and Tokatlidis, K. (2020). The biogenesis of mitochondrial intermembrane space proteins. J. Biol. Chem. 401: 737-747.

Elguindy, M.M., and Nakamaru-Ogiso, E. (2015). Apoptosis-inducing factor (AIF) and its family member protein, AMID, are rotenone- sensitive NADH:ubiquinone oxidoreductases (NDH-2). J. Biol. Chem. 290: 20815-20826.

Engel, T., Caballero-Caballero, A., Schindler, C.K., Plesnila, N., Strasser, A., Prehn, J.H., and Henshall, D.C. (2010). BH3-only protein Bid is dispensable for seizure-induced neuronal death and the associated nuclear accumulation of apoptosis-inducing factor. J. Neurochem. 115: 92-101.

Erdogan, A.J., Ali, M., Habich, M., Salscheider, S.L., Schu, L., Petrungaro, C., Thomas, L.W., Ashcroft, M., Leichert, L.I., Roma, L.P., et al. (2018). The mitochondrial oxidoreductase CHCHD4 is present in a semi-oxidized state in vivo. Redox Biol. 17: 200-206.

Finger, Y., and Riemer, J. (2020). Protein import by the mitochondrial disulfide relay in higher eukaryotes. J. Biol. Chem. 401: 749-763.

Fischer, M., Horn, S., Belkacemi, A., Kojer, K., Petrungaro, C., Habich, M., Ali, M., Kuttner, V., Bien, M., Kauff, F., et al. (2013). Protein import and oxidative folding in the mitochondrial intermembrane space of intact mammalian cells. Mol. Biol. Cell 24: 2160-2170.

Friederich, M.W., Erdogan, A.J., Coughlin, C.R., Il, Elos, M.T., Jiang, H., O'Rourke, C.P., Lovell, M.A., Wartchow, E., Gowan, K., Chatfield, K.C., et al. (2017). Mutations in the accessory subunit NDUFB10 result in isolated complex I deficiency and illustrate the critical role of intermembrane space import for complex I holoenzyme assembly. Hum. Mol. Genet. 26: 702-716.

Ghezzi, D., Sevrioukova, I., Invernizzi, F., Lamperti, C., Mora, M., D’Adamo, P., Novara, F., Zuffardi, O., Uziel, G., and Zeviani, M. (2010). Severe X-linked mitochondrial encephalomyopathy associated with a mutation in apoptosis-inducing factor. Am. J. Hum. Genet. 86: 639-649.

Gomes, F., Tahara, E.B., Busso, C., Kowaltowski, A.J., and Barros, M.H. (2013). nde1 deletion improves mitochondrial DNA maintenance in Saccharomyces cerevisiae coenzyme Q mutants. Biochem. J. 449: 595-603.

Gospodaryov, D.V., Strilbytska, O.M., Semaniuk, U.V., Perkhulyn, N.V., Rovenko, B.M., Yurkevych, I.S., Barata, A.G., Dick, T.P., Lushchak, O.V., and Jacobs, H.T. (2020). Alternative NADH dehydrogenase extends lifespan and increases resistance to xenobiotics in Drosophila. Biogerontology 21: 155-171.

Guida, M., Zanon, A., Montibeller, L., Lavdas, A.A., Ladurner, J., Pischedda, F., Rakovic, A., Domingues, F.S., Piccoli, G., Klein, C., et al. (2019). Parkin Interacts with apoptosis-inducing factor and interferes with its translocation to the nucleus in neuronal cells. Int. J. Mol. Sci. 20: 748.

Habich, M., Salscheider, S.L., Murschall, L.M., Hoehne, M.N., Fischer, M., Schorn, F., Petrungaro, C., Ali, M., Erdogan, A.J., Abou-Eid, S., et al. (2019). Vectorial Import via a metastable disulfide-linked complex allows for a quality control step and import by the mitochondrial disulfide relay. Cell Rep. 26: 759-774.e755.

Hangen, E., Blomgren, K., Benit, P., Kroemer, G., and Modjtahedi, N. (2010a). Life with or without AIF. Trends Biochem. Sci. 35: 278-287.

Hangen, E., De Zio, D., Bordi, M., Zhu, C., Dessen, P., Caffin, F., Lachkar, S., Perfettini, J.L., Lazar, V., Benard, J., et al. (2010b). A brain-specific isoform of mitochondrial apoptosis-inducing factor: AIF2. Cell Death Different. 17: 1155-1166.

Hangen, E., Feraud, O., Lachkar, S., Mou, H., Doti, N., Fimia, G.M., Lam, N.V., Zhu, C., Godin, I., Muller, K., et al. (2015). Interaction between AIF and CHCHD4 regulates respiratory chain biogenesis. Mol. Cell 58: 1001-1014.

Joza, N., Galindo, K., Pospisilik, J.A., Benit, P., Rangachari, M., Kanitz, E.E., Nakashima, Y., Neely, G.G., Rustin, P., Abrams, J.M., et al. (2008). The molecular archaeology of a 
mitochondrial death effector: AIF in Drosophila. Cell Death Different. 15: 1009-1018.

Joza, N., Susin, S.A., Daugas, E., Stanford, W.L., Cho, S.K., Li, C.Y., Sasaki, T., Elia, A.J., Cheng, H.Y., Ravagnan, L., et al. (2001). Essential role of the mitochondrial apoptosis-inducing factor in programmed cell death. Nature 410: 549-554.

Katsyuba, E., Mottis, A., Zietak, M., De Franco, F., van der Velpen, V., Gariani, K., Ryu, D., Cialabrini, L., Matilainen, O., Liscio, P., et al. (2018). De novo $\mathrm{NAD}^{+}$synthesis enhances mitochondrial function and improves health. Nature 563: 354-359.

Kollias, G., Kyriakopoulos, M., and Tiniakos, G. (1992). Epididymitis from Enterobius vermicularis: case report. J. Urol. 147: 1114-1116.

Li, W., Sun, L., Liang, Q., Wang, J., Mo, W., and Zhou, B. (2006). Yeast AMID homologue Ndi1p displays respiration-restricted apoptotic activity and is involved in chronological aging. Mol. Biol. Cell 17: 1802-1811.

Liu, L., Yang, C., Herzog, C., Seth, R., and Kaushal, G.P. (2010). Proteasome inhibitors prevent cisplatin-induced mitochondrial release of apoptosis-inducing factor and markedly ameliorate cisplatin nephrotoxicity. Biochem. Pharmacol. 79: 137-146.

Luttik, M.A., Overkamp, K.M., Kotter, P., de Vries, S., van Dijken, J.P., and Pronk, J.T. (1998). The Saccharomyces cerevisiae NDE1 and NDE2 genes encode separate mitochondrial NADH dehydrogenases catalyzing the oxidation of cytosolic NADH. J. Biol. Chem. 273: 24529-24534.

Marres, C.A., de Vries, S., and Grivell, L.A. (1991). Isolation and inactivation of the nuclear gene encoding the rotenone-insensitive internal NADH: ubiquinone oxidoreductase of mitochondria from Saccharomyces cerevisiae. Eur. J. Biochem. 195: 857-862.

Mate, M.J., Ortiz-Lombardia, M., Boitel, B., Haouz, A., Tello, D., Susin, S.A., Penninger, J., Kroemer, G., and Alzari, P.M. (2002). The crystal structure of the mouse apoptosis-inducing factor AIF. Nat. Struct. Mol. Biol. 9: 442-446.

Matus-Ortega, M.G., Salmeron-Santiago, K.G., Flores-Herrera, O., Guerra-Sanchez, G., Martinez, F., Rendon, J.L., and Pardo, J.P. (2011). The alternative NADH dehydrogenase is present in mitochondria of some animal taxa. Comp. Biochem. Physiol. Part D Genomics Proteomics 6: 256-263.

McDonald, A.E., and Gospodaryov, D.V. (2019). Alternative NAD(P)H dehydrogenase and alternative oxidase: proposed physiological roles in animals. Mitochondrion 45: 7-17.

Mesecke, N., Terziyska, N., Kozany, C., Baumann, F., Neupert, W., Hell, K., and Herrmann, J.M. (2005). A disulfide relay system in the intermembrane space of mitochondria that mediates protein import. Cell 121: 1059-1069.

Meyer, K., Buettner, S., Ghezzi, D., Zeviani, M., Bano, D., and Nicotera, P. (2015). Loss of apoptosis-inducing factor critically affects MIA40 function. Cell Death Dis. 6: e1814.

Miramar, M.D., Costantini, P., Ravagnan, L., Saraiva, L.M., Haouzi, D., Brothers, G., Penninger, J.M., Peleato, M.L., Kroemer, G., and Susin, S.A. (2001). NADH oxidase activity of mitochondrial apoptosis-inducing factor. J. Biol. Chem. 276: 16391-16398.

Mokranjac, D. (2020). How to get to the other side of the mitochondrial inner membrane - the protein import motor. J. Biol. Chem. 401: 723-736.

Moubarak, R.S., Yuste, V.J., Artus, C., Bouharrour, A., Greer, P.A., Menissier-de Murcia, J., and Susin, S.A. (2007). Sequential activation of poly(ADP-ribose) polymerase 1 , calpains, and Bax is essential in apoptosis-inducing factor-mediated programmed necrosis. Mol. Cell. Biol. 27: 4844-4862.
Muzaffar, S., and Chattoo, B.B. (2017). Apoptosis-inducing factor (Aif1) mediates anacardic acid-induced apoptosis in Saccharomyces cerevisiae. Apoptosis 22: 463-474.

Nguyen, H.P., Yi, D., Lin, F., Viscarra, J.A., Tabuchi, C., Ngo, K., Shin, G., Lee, A.Y., Wang, Y., and Sul, H.S. (2020). Aifm2, a NADH oxidase, supports robust glycolysis and is required for cold- and dietinduced thermogenesis. Mol. Cell 77: 600-617. e604.

Norberg, E., Gogvadze, V., Vakifahmetoglu, H., Orrenius, S., and Zhivotovsky, B. (2010a). Oxidative modification sensitizes mitochondrial apoptosis-inducing factor to calpain-mediated processing. Free Radical Biol. Med. 48: 791-797.

Norberg, E., Orrenius, S., and Zhivotovsky, B. (2010b). Mitochondrial regulation of cell death: processing of apoptosis-inducing factor (AIF). Biochem. Biophys. Res. Commun. 396: 95-100.

Okamoto, H., Miyagawa, A., Shiota, T., Tamura, Y., and Endo, T. (2014). Intramolecular disulfide bond of Tim22 protein maintains integrity of the TIM22 complex in the mitochondrial inner membrane. J. Biol. Chem. 289: 4827-4838.

Otera, H., Ohsakaya, S., Nagaura, Z., Ishihara, N., and Mihara, K. (2005). Export of mitochondrial AIF in response to proapoptotic stimuli depends on processing at the intermembrane space. EMBO J. 24: 1375-1386.

Ozaki, T., Yamashita, T., and Ishiguro, S. (2008). ERp57-associated mitochondrial mu-calpain truncates apoptosis-inducing factor. Biochim. Biophys. Acta 1783: 1955-1963.

Ozaki, T., Yamashita, T., and Ishiguro, S. (2009). Mitochondrial $\mathrm{m}$-calpain plays a role in the release of truncated apoptosisinducing factor from the mitochondria. Biochim. Biophys. Acta 1793: 1848-1859.

Peleh, V., Cordat, E., and Herrmann, J.M. (2016). Mia40 is a trans-site receptor that drives protein import into the mitochondrial intermembrane space by hydrophobic substrate binding. eLife 5, https://doi.org/10.7554/elife.16177.

Peleh, V., Zannini, F., Backes, S., Rouhier, N., and Herrmann, J.M. (2017). Erv1 of Arabidopsis thaliana can directly oxidize mitochondrial intermembrane space proteins in the absence of redox-active Mia40. BMC Biol. 15: 106.

Pena-Blanco, A., and Garcia-Saez, A.J. (2018). Bax, Bak and beyond mitochondrial performance in apoptosis. FEBS J. 285: 416-431.

Petrungaro, C., Zimmermann, K.M., Kuttner, V., Fischer, M., Dengjel, J., Bogeski, I., and Riemer, J. (2015). The $\mathrm{Ca}^{2+}$-dependent release of the Mia40-Induced MICU1-MICU2 dimer from MCU regulates mitochondrial $\mathrm{Ca}^{2+}$ uptake. Cell Metab. 22: 721-733.

Polster, B.M., Basanez, G., Etxebarria, A., Hardwick, J.M., and Nicholls, D.G. (2005). Calpain I induces cleavage and release of apoptosis-inducing factor from isolated mitochondria. J. Biol. Chem. 280: 6447-6454.

Ramesh, A., Peleh, V., Martinez-Caballero, S., Wollweber, F., Sommer, F., van der Laan, M., Schroda, M., Alexander, R.T., Campo, M.L., and Herrmann, J.M. (2016). A disulfide bond in the TIM23 complex is crucial for voltage gating and mitochondrial protein import. J. Cell Biol. 214: 417-431.

Riemer, J., Schwarzlander, M., Conrad, M., and Herrmann, J.M. (2015). Thiol switches in mitochondria: operation and physiological relevance. J. Biol. Chem 396: 465-482.

Rinaldi, C., Grunseich, C., Sevrioukova, I.F., Schindler, A., HorkayneSzakaly, I., Lamperti, C., Landoure, G., Kennerson, M.L., Burnett, B.G., Bonnemann, C., et al. (2012). Cowchock syndrome is associated with a mutation in apoptosis-inducing factor. Am. J. Hum. Genet. 91: 1095-1102. 
Rissler, M., Wiedemann, N., Pfannschmidt, S., Gabriel, K., Guiard, B., Pfanner, N., and Chacinska, A. (2005). The essential mitochondrial protein Erv1 cooperates with Mia40 in biogenesis of intermembrane space proteins. J. Mol. Biol. 353: 485-492.

Rossmann, M.G., and Argos, P. (1978). The taxonomy of binding sites in proteins. Mol. Cell. Biochem. 21: 161-182.

Saladi, S., Boos, F., Poglitsch, M., Meyer, H., Sommer, F., Muhlhaus, T., Schroda, M., Schuldiner, M., Madeo, F., and Herrmann, J.M. (2020). The NADH dehydrogenase Nde1 executes cell death after integrating signals from metabolism and proteostasis on the mitochondrial surface. Mol. Cell 77: 189-202.

Sorrentino, L., Calogero, A.M., Pandini, V., Vanoni, M.A., Sevrioukova, I.F., and Aliverti, A. (2015). Key role of the adenylate moiety and integrity of the adenylate-binding site for the $\mathrm{NAD}^{+} / \mathrm{H}$ binding to mitochondrial apoptosis-inducing factor. Biochemistry 54 : 6996-7009.

Sorrentino, L., Cossu, F., Milani, M., Aliverti, A., and Mastrangelo, E. (2017). Structural bases of the altered catalytic properties of a pathogenic variant of apoptosis inducing factor. Biochem. Biophys. Res. Commun. 490: 1011-1017.

Susin, S.A., Lorenzo, H.K., Zamzami, N., Marzo, I., Snow, B.E., Brothers, G.M., Mangion, J., Jacotot, E., Costantini, P., Loeffler, M., et al. (1999). Molecular characterization of mitochondrial apoptosis-inducing factor. Nature 397: 441-446.

Susin, S.A., Zamzami, N., Castedo, M., Hirsch, T., Marchetti, P., Macho, A., Daugas, E., Geuskens, M., and Kroemer, G. (1996). $\mathrm{Bcl}-2$ inhibits the mitochondrial release of an apoptogenic protease. J. Exp. Med. 184: 1331-1341.

Thompson, J., Hu, Y., Lesnefsky, E.J., and Chen, Q. (2016). Activation of mitochondrial calpain and increased cardiac injury: beyond AIF release. Am. J. Physiol. Heart Circulat. Physiol. 310: H376-H384.

Thompson Legault, J., Strittmatter, L., Tardif, J., Sharma, R., TremblayVaillancourt, V., Aubut, C., Boucher, G., Clish, C.B., Cyr, D., Daneault, C., et al. (2015). A metabolic signature of mitochondrial dysfunction revealed through a monogenic form of Leigh syndrome. Cell Rep. 13: 981-989.

Troulinaki, K., Buttner, S., Marsal Cots, A., Maida, S., Meyer, K., Bertan, F., Gioran, A., Piazzesi, A., Fornarelli, A., Nicotera, P., et al. (2018). WAH-1/AIF regulates mitochondrial oxidative phosphorylation in the nematode Caenorhabditis elegans. Cell Death Discov. 4: 2.

Urbano, A., Lakshmanan, U., Choo, P.H., Kwan, J.C., Ng, P.Y., Guo, K., Dhakshinamoorthy, S., and Porter, A. (2005). AIF suppresses chemical stress-induced apoptosis and maintains the transformed state of tumor cells. EMBO J. 24: 2815-2826.

Vahsen, N., Cande, C., Briere, J.J., Benit, P., Joza, N., Larochette, N., Mastroberardino, P.G., Pequignot, M.O., Casares, N., Lazar, V., et al. (2004). AlF deficiency compromises oxidative phosphorylation. EMBO J. 23: 4679-4689.

Wischhof, L., Gioran, A., Sonntag-Bensch, D., Piazzesi, A., Stork, M., Nicotera, P., and Bano, D. (2018). A disease-associated Aifm1 variant induces severe myopathy in Knockin mice. Mol. Metab. 13: 10-23.

Wissing, S., Ludovico, P., Herker, E., Buttner, S., Engelhardt, S.M., Decker, T., Link, A., Proksch, A., Rodrigues, F., Corte-Real, M., et al. (2004). An AIF orthologue regulates apoptosis in yeast. J. Cell Biol. 166: 969-974.
Wrobel, L., Sokol, A.M., Chojnacka, M., and Chacinska, A. (2016). The presence of disulfide bonds reveals an evolutionarily conserved mechanism involved in mitochondrial protein translocase assembly. Sci. Rep. 6: 27484.

Yu, S.W., Wang, Y., Frydenlund, D.S., Ottersen, O.P., Dawson, V.L., and Dawson, T.M. (2009). Outer mitochondrial membrane localization of apoptosis-inducing factor: mechanistic implications for release. ASN Neuro. 1: e00021.

Zahedi, R.P., Sickmann, A., Boehm, A.M., Winkler, C., Zufall, N., Schonfisch, B., Guiard, B., Pfanner, N., and Meisinger, C. (2006). Proteomic analysis of the yeast mitochondrial outer membrane reveals accumulation of a subclass of preproteins. Mol. Biol. Cell 17: 1436-1450.

Zong, L., Guan, J., Ealy, M., Zhang, Q., Wang, D., Wang, H., Zhao, Y., Shen, Z., Campbell, C.A., Wang, F., et al. (2015). Mutations in apoptosis-inducing factor cause $\mathrm{X}$-linked recessive auditory neuropathy spectrum disorder. J. Med. Genet. 52: 523-531.

\section{Bionotes}

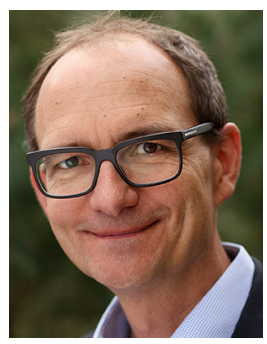

Johannes M. Herrmann

Department of Cell Biology, University of Kaiserslautern, Erwin-Schrödinger-Strasse 13, D-67663 Kaiserslautern, Germany hannes.herrmann@biologie.uni-kl.de https://orcid.org/0000-0003-2081-4506

Johannes M. Herrmann is Professor for Cell Biology at the University in Kaiserslautern since 2006. He studied Biology and Biochemistry in Bayreuth and Tübingen. After his PhD with Walter Neupert in Munich, he worked as a postdoc with Randy Schekman at the University of California in Berkeley. His main research field is the biogenesis of mitochondria.

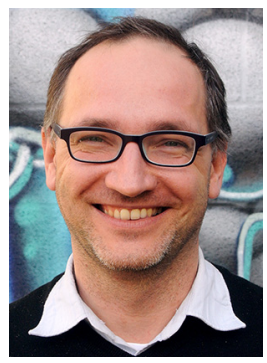

\section{Jan Riemer}

Department of Biochemistry, University of Cologne, Zülpicher Str. 47A, D-50674

Cologne, Germany

jan.riemer@uni-koeln.de

Jan Riemer is Professor for Biochemistry at the University of Cologne since 2015. He studied Biochemistry in Tübingen and Ann Arbor. After his PhD with Lars Ellgaard in Zurich and Copenhagen, he worked as group leader and Assistant Professor in Kaiserslautern before moving to Cologne. His main research field is mitochondrial biogenesis and redox regulation of cellular processes. 